\title{
Patient-reported outcomes with golimumab in patients with rheumatoid arthritis, psoriatic arthritis, and ankylosing spondylitis: non-interventional study GO-NICE in Germany
}

\author{
Klaus Krüger ${ }^{1} \cdot$ Gerd R. Burmester $^{2}$. Siegfried Wassenberg ${ }^{3} \cdot$ Martin Bohl-Bühler $^{4} \cdot$ Matthias H. Thomas $^{5}$
}

Received: 9 September 2018 / Accepted: 15 October 2018 / Published online: 10 November 2018

(c) The Author(s) 2018

\begin{abstract}
The TNF inhibitor golimumab (GLM) is a treatment option in patients with rheumatoid arthritis (RA), psoriatic arthritis (PsA), and ankylosing spondylitis (AS). The GO-NICE study assessed patient-reported outcomes (PRO) in patients newly treated with monthly GLM $50 \mathrm{mg}$ subcutaneously (SC) under real-life conditions in Germany. A prospective non-interventional study with 24-month observation per patient was conducted at 158 sites. Available for analysis were 1,458 patients, 474 with rheumatoid arthritis (RA: $54.9 \pm 13.4$ years, $72.8 \%$ females, $60.4 \%$ biologic-naïve), 501 with psoriatic arthritis (PsA: $50.5 \pm 12.1$ years, $54.1 \%$ females; $47.5 \%$ biologic-naïve), and 483 with ankylosing spondylitis (AS: $43.6 \pm 12.3$ years, $66.5 \%$ males; $58.4 \%$ biologic-naïve). A total of 664 patients completed follow-up to month 24 . An improvement of QoL by EuroQoL EQ-5D-3L was seen after 6 months and was maintained over 24 months. The patients' health state today (EQ visual analog scale) improved statistically significantly ( $p<0.0001 \mathrm{vs.} \mathrm{BL)} \mathrm{from} 51.0$ at baseline (BL) to 63.4 (RA), from 48.4 to 64.3 (PsA) and from 46.8 to 66.5 (AS). Functional ability (FFbH) improved significantly ( $p<0.003$ vs. BL) from BL 68.2 to 76.1 points (RA), from 69.0 to 76.8 points (PsA), and from 69.0 to 78.5 points (AS). The mean FACIT-Fatigue score increased significantly ( $p<0.0001 \mathrm{vs.} \mathrm{BL)}$ from BL 32.4 to 38.3 points (RA), from 30.0 to 35.9 points (PsA), and from 29.9 to 37.9 points after 24 months (AS); $p<0.0001$ vs. BL each. On treatment with GLM SC once monthly, significant improvements in patient-reported QoL parameters were noted in a very similar manner in all three diseases.

Trial registration ClinTrials.gov Identifier: NCT01313858. Registered March 14, 2011; https://clinicaltrials.gov/ct2/show/ record/NCT01313858.
\end{abstract}

Keywords Golimumab · Rheumatoid arthritis · Psoriatic arthritis · Ankylosing spondylitis · Patient reported outcomes · Non-interventional study

\section{Abbreviations}

AS

Ankylosing spondylitis

EULAR European League Against Rheumatism

FACIT-F Functional Assessment of Chronic Illness Therapy Fatigue

$\mathrm{FFbH} \quad$ Functional ability

$\begin{array}{ll}\text { GLM } & \text { Golimumab } \\ \text { PRO } & \text { Patient-reported outcomes } \\ \text { PsA } & \text { Psoriatic arthritis } \\ \text { QoL } & \text { Quality of life } \\ \text { RA } & \text { Rheumatoid arthritis }\end{array}$

Rheumatologisches Praxiszentrum, Munich, Germany

2 Department of Rheumatology and Clinical Immunology, Cha rité-Universitätsmedizin, Berlin, Germany

3 Rheumazentrum, Ratingen, Germany

4 Rheumahaus Potsdam GbR, Potsdam, Germany

5 Medical Affairs, MSD Sharp \& Dohme GmbH, Lindenplatz 1, 85540 Haar, Germany

Siegfried Wassenberg

swassenberg@clinic.de

Martin Bohl-Bühler

Info@Rheumahaus.de 
SC Subcutaneous

TNFi Tumour necrosis factor inhibitors

\section{Background}

Patient-related outcomes (PRO) are defined as reports provided by patients about their own health, quality of life, or functional status associated with the health care or treatment they have received [1]. The importance of including the patient perspective by assessing PROs in clinical trials has been emphasized, among others, by major international health policy and regulatory authorities $[2,3]$.

Rheumatic diseases in general lead to pain, joint damage, fatigue, and reduced activities of daily living and mobility [4]. Therefore, quality of life (QoL) and other PRO measures have received high attention in this indication [5-7]. The European League Against Rheumatism (EULAR) emphasizes that patient perspectives and priorities in treatment decisions are an overarching principle of care for rheumatoid arthritis (RA) patients [8].

Rheumatoid arthritis, (RA) and the spondyloarthropathies psoriatic arthritis (PsA) and ankylosing spondylitis (AS) - as three common immune-mediated rheumatic diseases-have different phenotypes but share important pathophysiologic mechanisms. Also, they are tackled with similar treatment approaches with anti-inflammatory medications, including non-steroidal anti-inflammatory drugs, glucocorticoids, disease-modifying anti-rheumatic drugs (DMARDs), and biologics including tumour necrosis factor inhibitors (TNFi). The newer biological agents have been reported to have a beneficial impact on health-related QoL and productivity in RA [9].

Golimumab (GLM) is one of the newer, second-generation TNF inhibitors [10]. It is injected once a month subcutaneously, permitting self-injection and thus greater flexibility and convenience for patients. The efficacy and safety of GLM has been shown in these indications in a number of large-scale randomised controlled trials (RCT) [11-14], and their open-label 5-year extensions [15-18]. These studies have entered selected patients in terms of disease characteristics, comorbidities and concomitant medications, and have been performed nearly a decade ago [19].

Data on the effect of GLM on the patient-reported outcomes (PROs) quality of life (QoL), fatigue, and functionality in daily clinical practice are lacking.

In a previous analysis, the effectiveness and safety of GLM $50 \mathrm{mg}$ SC once monthly was shown in patients with RA, PsA, and AS in a real-life setting in Germany. In that study, Disease Activity Score 28-joint count erythrocyte sedimentation rate (DAS28-ESR) decreased from 5.0 to 2.9 after 24 months $(p<0.0001)$ in patients with RA, and Bath Ankylosing Spondylitis Disease Index score decreased from
5.1 to 2.4 ( $p<0.0001)$ in patients with AS. Response rate calculated in patients with PsA by modified Psoriatic Arthritis Response Criteria was $67.9 \%$ after 24 months. Thus, during the 24-month observation periods substantial improvement in disease activity (DAS28 and BASDAI), response (PsARC) was seen early at 3 months and was maintained throughout the 24-month observation period in line with the previous clinical studies [20].

Thus, the present analysis of the pragmatic GO-NICE study aims to describe the effects of GLM treatment from the patient perspective using PROs in the indications RA, PsA, and AS, under daily practice conditions.

\section{Methods}

\section{Study design}

GO-NICE is an open-label, multicenter, prospective observational study, which was performed between 2010 and 2015 at 158 German sites, mostly by rheumatologists $(92.4 \%$ of the physicians). Details on the study design, patient characteristics, the clinical outcomes, and safety data have been reported earlier [20].

Patients were managed according to the treating physician's clinical judgment and without any protocol-defined therapeutic or diagnostic procedures. In addition, no inclusion and exclusion criteria were specified in contrast to interventional studies as observational studies are open to all patients eligible to receive treatment according to the prescribing information [21].

\section{Patients}

Adult patients were eligible if they met the inclusion criteria: diagnosis of RA, PsA or AS; absence of any contraindication for GLM; patient consent for participation; no prior treatment with GLM and indication for use of GLM applied subcutaneously (SC) with an auto-injector at a dosage of $50 \mathrm{mg}$ as specified in the product labelling [22]. There were no explicit exclusion criteria to avoid patient selection bias. All patients were treated with GLM $50 \mathrm{mg}$ SC according to the Summary of Product Characteristics (SPC). Patients were evaluated prior to the first administration of GLM and in 3-monthly intervals thereafter over a 2-year period.

\section{Physician assessments}

Physician Global Assessment (PhGA) is a non-diseasespecific evaluation of participants' overall health status assessed on a visual analogue scale (VAS) ranging from "0" (free of complaints) to "10" (strong discomfort). The closer the score is to 0 , the better the health status. PhGA 
was assessed in all three groups by the treating physician on a VAS ranging from $0=$ free of complaints to $10=$ strong discomfort.

\section{PRO instruments and assessments}

\section{EuroQoL}

General healthcare evaluation was done with the EuroQoL five-dimensional questionnaire (EQ-5D-3L). It consists of five domains (mobility, self-care, usual activities, pain/discomfort, and anxiety/depression); each is divided into three levels of perceived problems ("no, some or extreme problems"). In addition, patients rate their current health on a 20 -cm vertical visual analogue scale (VAS) scored from 0 to 100 reflecting the continuum from the best imaginable to the worst imaginable health state. The EQ-5D-3L is validated for the German population [23].

\section{Hannover functional ability questionnaire}

The $\mathrm{FFbH}$ is a participant questionnaire assessing disability/ functional impairment. Ability to perform 18 activities of daily living are scored on a 3 -point scale $(2=$ Yes, $1=$ Yes but with effort, and $0=$ No or with assistance) and summed. Remaining functional capacity is calculated as the percent of the maximum number of score points $(\mathrm{FFbH}=$ (Attained score $\times 100) /(2 \times n)$ where $\mathrm{n}$ is the number of completed responses) with range from $0=$ total loss of functional capacity to $100=$ maximal functional capacity. Increase from baseline in $\mathrm{FFbH}$ score signifies improvement. The $\mathrm{FFbH}$ is similar to Health Assessment Questionnaire (HAQ) but is more widely used in Germany, and both show a high degree of correspondence, as a validation study in RA patients in Germany has shown [24].

\section{Fatigue}

Fatigue was assessed with the Functional Assessment of Chronic Illness Therapy Fatigue (FACIT-F) questionnaire, originally developed for cancer, in which patients score (0-52) tiredness, weakness, and difficulty with usual activities because of fatigue (increased score indicates reduced fatigue) [25]. The questionnaire was validated in a PsA cohort in a Canadian study, showing high internal consistency, test-retest reliability, and criterion and construct validity [26]. Also, it was responsive to change in a trial of adalimumab in PsA [27]. Reliability and validity of the German translation of the FACIT-Fatigue Scale were confirmed recently [28].

\section{Data management and statistics}

Investigators or their staff used an online electronic data capture system to enter clinical parameters into the database. Patients provided PRO data on paper forms, which were entered into the database by trained staff of a contract research organisation. In a subset of centres, study data were compared with the patient files (source data verification).

Analyses were performed in an exploratory manner using descriptive statistical methods. All effectiveness analyses were conducted for the evaluable patients (who had the baseline (BL) assessment and at least one additional visit) and the completer patients (who had the BL assessment and the visit at month 24), grouped by indication [20]. For continuous variables, the number of patients with non-missing data, mean, $\mathrm{SD}$, minimum, $25 \%$ quartile, median, $75 \%$ quartile and maximum were calculated. For ordinal and categorical variables, frequencies were calculated based on all observations with non-missing data for this variable. Incomplete datasets were included in the analysis. Missing GLM treatment start date was replaced by date of visit 1 . There was no imputation of missing values for any endpoint. Changes in variables by time were calculated with least square means, standard error of the man, and $95 \%$ confidence intervals. P-values were calculated with chi square or Wilcoxon tests, for PhGA with signed rank test. No sensitivity analyses were performed. PRO data were analysed based on the instructions of the respective tool developers.

\section{Results}

\section{Patient disposition}

A total of 1458 patients had a baseline assessment as well as at least one additional visit and were eligible for final analysis (evaluable population): 474 (32.5\%) with RA, 501 (34.4\%) with PsA, and 483 (33.1\%) with AS. The 24-month observational period was completed by 664 patients (45.5\%). Details are shown in Fig. 1.

\section{Baseline characteristics of the evaluable population}

At baseline (BL), mean age of patients was lowest in the patients with AS (43.6 \pm 12.3 years) compared to those with PsA $(50.3 \pm 12.1$ years $)$ and RA $(54.9 \pm 13.4$ years, Table 1$)$. The proportion of females was lower in AS (33.5\%) than in PsA $(54.1 \%)$ and RA $(72.8 \%)$.

In line with the age pattern, full-time employment was highest in patients with AS (62.1\%) compared to those with PsA (45.1\%) and RA (32.6\%).

Mean disease duration since initial diagnosis was 10.4 years in RA, 13.0 years in PsA, and 9.8 years in AS. In 
Fig. 1 The flow chart shows the patient disposition and flow during the study. Of the 1613 documented patients (safety set), 1458 were eligible for the efficacy analysis (efficacy set), and 664 completed the study (completer set). $A S$ ankylosing spondylitis, $B L$ baseline, $P s A$ psoriatic arthritis, $R A$ rheumatoid arthritis

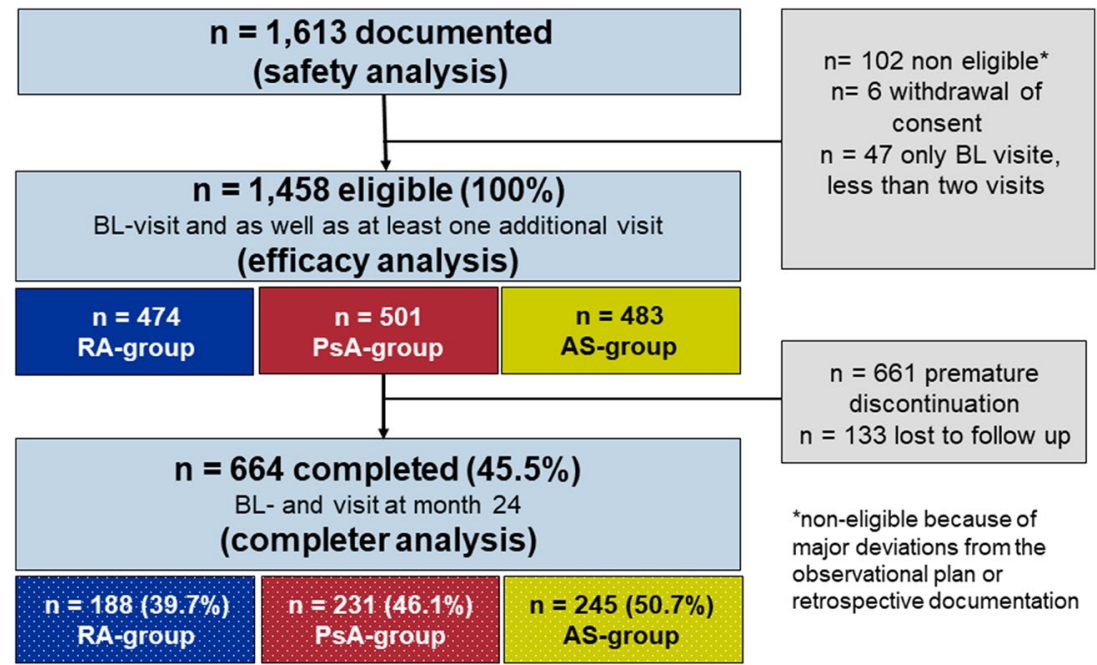

Table 1 Baseline characteristics

\begin{tabular}{llll}
\hline & RA $(n=474)$ & PsA $(n=501)$ & AS $(n=483)$ \\
\hline Demographics & & & \\
Age, years & $54.9 \pm 13.4$ & $50.5 \pm 12.1$ & $43.6 \pm 12.3$ \\
$\quad$ Range & $19-82$ & $18-83$ & $18-73$ \\
Males, $n$ (\%) & $129(27.2)$ & $230(45.9)$ & $321(66.5)$ \\
Mean Body Mass Index, kg/m ${ }^{2}$ & $26.5 \pm 4.9$ & $28.1 \pm 5.4$ & $26.7 \pm 5.5$ \\
Time since first diagnosis, years & $10.4 \pm 8.9$ & $13.0 \pm 11.5$ & $9.8 \pm 9.4$ \\
At least one concomitant disease & $264(55.7)$ & $258(51.5)$ & $203(42.0)$ \\
Patients with extra-articular manifestations & $73(15.4)$ & $439(87.8)$ & $163(33.9)$ \\
(Pre-)treatment status & & & \\
Biologic-naïve, $n$ (\%) & $305(64.3)$ & $286(57.1)$ & $292(60.5)$ \\
Patients currently on NSAIDs, coxibs, analgesics & $258(54.4)$ & $333(66.5)$ & $416(86.2)$ \\
Patients currently on basic therapy or immunosuppressants & $407(85.8)$ & $322(64.3)$ & $133(27.6)$ \\
Patients currently on syst. glucocorticoids & $360(75.9)$ & $206(41.1)$ & $22(4.6)$ \\
Employment status & & & \\
Full time, $n$ (\%) & $154(32.6)$ & $225(45.1)$ & $298(62.1)$ \\
Part time, $n(\%)$ & $64(13.1)$ & $53(10.6)$ & $36(7.5)$ \\
Unemployed, $n$ (\%) & $24(5.1)$ & $50(10.0)$ & $52(10.8)$ \\
Housewife/houseman, $n$ (\%) & $51(10.8)$ & $30(6.0)$ & $16(3.3)$ \\
Pupil/ student/ in apprenticeship, $n$ (\%) & $9(1.9)$ & $15(3.0)$ & $11(2.3)$ \\
Early pension, $n$ (\%) & $29(6.1)$ & $46(9.2)$ & $21(4.4)$ \\
Old-age pension, $n$ (\%) & $128(27.1)$ & $63(12.6)$ & $29(6.0)$ \\
Handicapped (unable to work in his/her Profession or work & $15(3.2)$ & $17(3.4)$ & $17(3.5)$ \\
at all), $n(\%)$ & & & \\
\hline
\end{tabular}

Values are mean \pm standard deviations, or number of patients (percentages), respectively terms of treatment history, patients without any prior biologics use were $64.3 \%$ in RA, $57.1 \%$ in PsA, and $60.5 \%$ in AS.

\section{Physician global assessment}

In patients with RA the mean PhGA on the 10-point VAS improved significantly from 5.7 at $\mathrm{BL}$ to $3.4(-2.3)$ at month 3 and to $2.2(-3.5)$ at month 24 , in patients with
PsA from 5.5 at BL to $3.2(-2.3)$ at month 3 , and to 2.1 ( -3.4 ) at month 24 , and in patients with AS from 5.7 at BL to $2.9(-2.8)$ at month 3 , and to $2.1(-3.6)$ at month 24 ( $p<0.0001$ vs. BL, each) (Fig. 2). 
Fig. 2 Physician Global Assessment (PhGA) of patient's health status. The PhGA is a nondisease-specific evaluation of participants' overall health status assessed on a 10-mm visual analogue scale (VAS) ranging from " 0 " (free of complaints) to "10" (strong discomfort). The closer the score is to 0 , the better is the health status

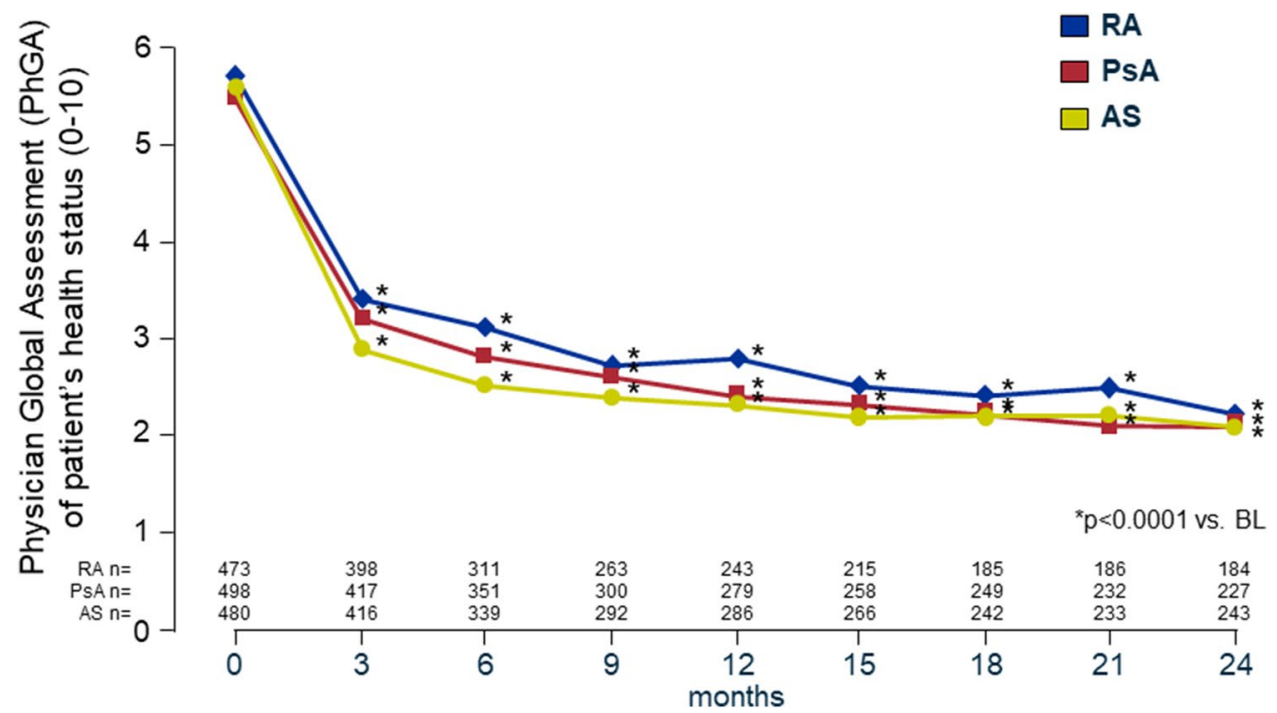

\section{Patient-reported outcomes}

\section{QoL by EQ VAS}

Patients' self-reported health state by EQ VAS improved in all indications, and the effect was maintained throughout the observation (Fig. 3).

The patients' health state today (EQ VAS) improved significantly ( $p<0.0001$ vs. BL, each) from 51.0 at baseline (BL) to $63.4(+12.4)(\mathrm{RA})$, from 48.4 to $64.3(+15.9)$ (PsA), and from 46.8 to 66.5 (+19.7) (AS) during the course of treatment within 24 months.

The benefit was most pronounced within the first 6 months in all disease groups.

\section{EQ-5D-3L}

Patients most frequently reported impairments in the domain pain/discomfort, followed by usual activities, mobility, anxiety/depression, and self-care. In all disease groups, substantial improvements (all but one statistically significant) were noted during follow-up compared to BL. Effects occurred early and were maintained throughout the study (Fig. 4a-c).

\section{Functional ability (FFbH)}

An improvement in functional ability by $\mathrm{FFbH}$ was also shown during the 24 months of therapy. The mean $\mathrm{FFbH}$ score increased significantly from baseline 68.7 to 74.0 $(+5.3)$ at month $3(p<0.001)$ and $76.1(+7.4)$ at month 24

Fig. 3 EQ visual analogue scale (EQ VAS) from $0=$ 'worst imaginable health state' to 100 $=$ 'best imaginable health state'. $B L$ baseline

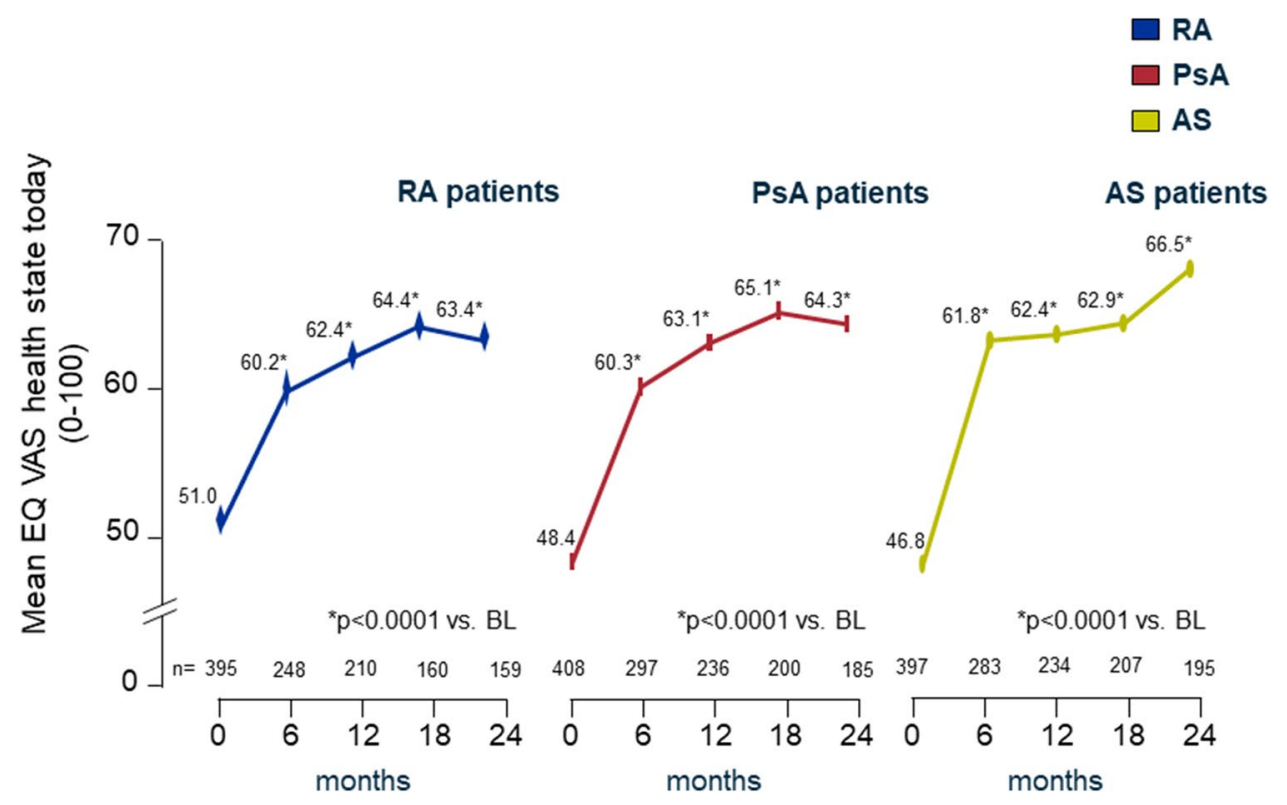


Fig. 4 EQ-5D-3L: Euro-QoL descriptive system of healthrelated quality of life states consisting of five dimensions and three levels. The figure shows the proportion of patients reporting some or extreme problems, by visit
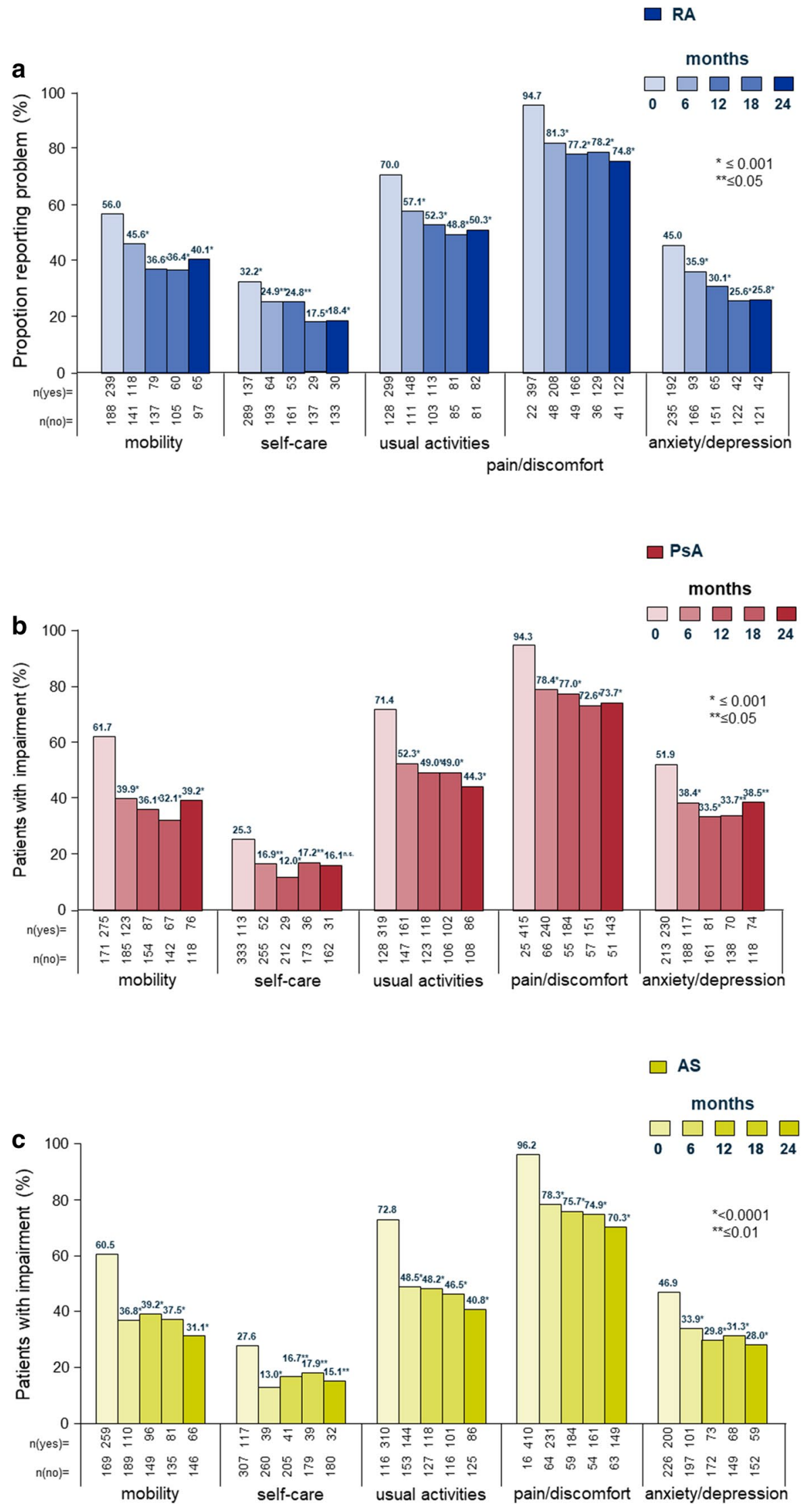
in patients with RA, from $69.0 \%$ to $74.9(+5.9)$ at month 3 $(p<0.0002)$ and $76.8(+7.8)$ at month 24 in patients with PsA, and from 69.0to 77.7 $(+5.9)$ at month $3(p<0.0001)$ and 78.5(+9.5) at month 24 in patients with AS (Fig. 5).

\section{Functional assessment of chronic illness therapy: fatigue (FACIT-F)}

The mean FACIT-Fatigue score increased significantly $(p<0.0001)$ in patients with RA from baseline 32.4 to 35.8 $(+3.4)$ at month 3 and to $38.3(+5.9)$ points at month 24 , in patients with PsA from 30.0 to $34.4(+4.4)$ at month 3 and to $35.9(+5.9)$ points at month 24 , and in patients with AS from $29.9(+5.2)$ to 35.1 at month 3 and to $37.9(+8.0)$ points at month 24 (Fig. 6).

\section{Safety}

There were no previously unknown adverse drug effects detected in the study.

\section{Discussion}

The present study is the first to report real-world data on PRO on the three main inflammatory rheumatic diseases RA, PsA, and AS for which GLM is approved. GLM administered in the $50 \mathrm{mg}$ dose subcutaneously in monthly intervals was an effective treatment in patients with RA, PsA, and AS in a real-life setting in Germany. During the 2-year observation, substantial improvements, patient-reported quality of
Fig. 5 FFbH: Funktionsfragebogen Hannover. 0 represents minimal function, 100 optimal function
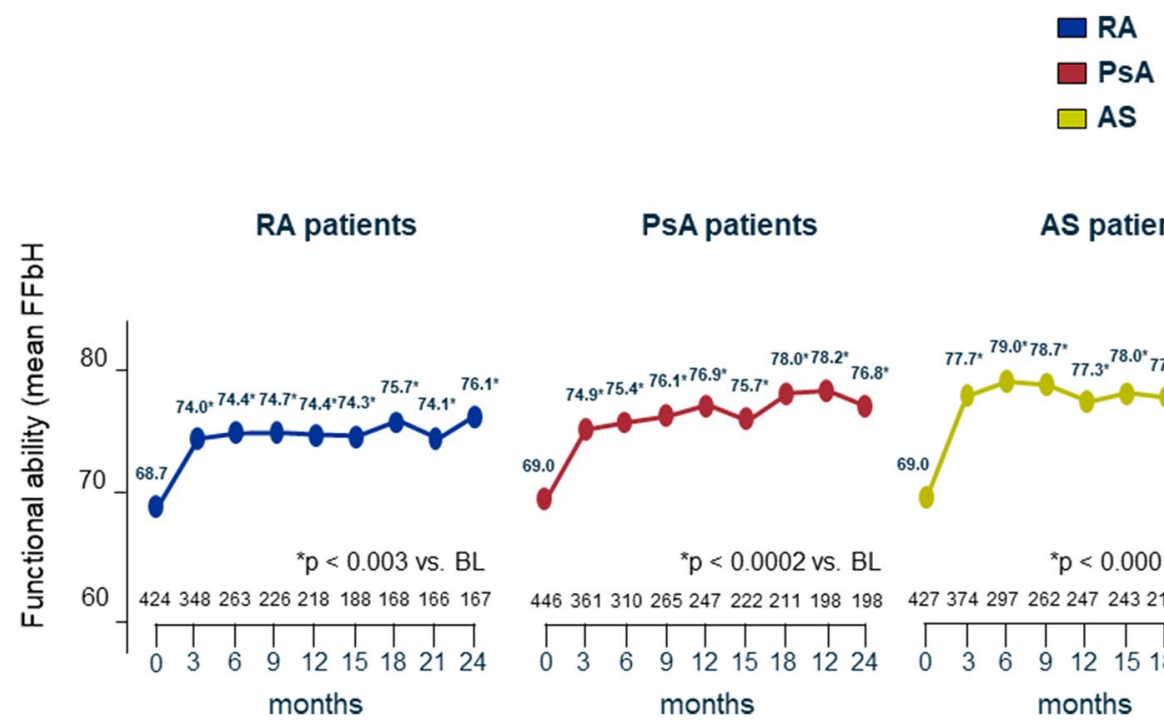

PsA patients

AS patients
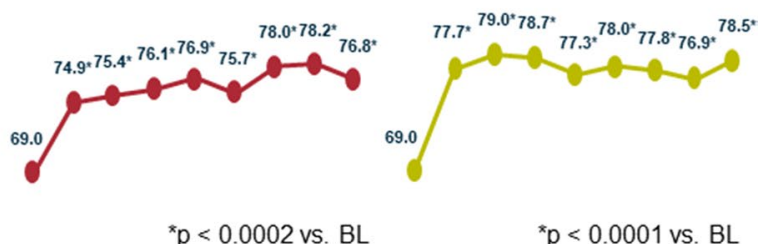

446361310265247222211198198

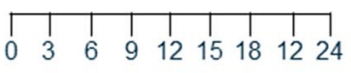
months

427374297262247243216200211

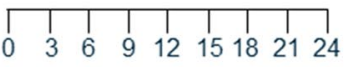

months

Fig. 6 FACIT-Fatigue: functional assessment of chronic illness therapy-fatigue. On the FACIT-F, 0 points represent the worst and 52 the highest possible state with regard to fatigue 
life, functional capacity, and fatigue were seen. The study complements the findings of various phase III controlled studies which were the basis for regulatory approval [19].

The mentioned randomised controlled studies reported limited health-related QoL data. In the RA GO-AFTER study, only FACIT-F and visual analogue scales for pain or overall disease activity were used, but no instrument for generic QoL assessment [29]. Also the pivotal RA studies GO-BEFORE and GO-FORWARD have not presented health-related QoL data $[11,30]$. In AS GO-RAISE study, the Short-Form (SF-) 36 [31] was used and the physical and mental components showed clear improvement [13, 32]. In the PsA GO-REVEAL study, the physical component of the SF-36 was also improved [12].

The present study adds, for the first time, data on the EQ-5D-3L and EQ VAS, over 2 years' follow-up. Effects of GLM treatment on QoL, fatigue, and functional ability were substantial and were maintained over the full observation period.

The $\mathrm{FFbH}$ has been shown earlier to be a reliable and valid instrument for measuring functional disability in a German-speaking population with RA [24] In all groups in GO-NICE, scores increased in a clinically relevant manner by the end of the study. They were higher compared to a recent large observational study in RA with another biologic which used the $\mathrm{FFbH}$ as primary endpoint (mean score at 1 year was approximately 65 points) [33].

Fatigue has increasingly been recognized as an important clinical dimension by patients with rheumatic diseases [34]. In a recent international survey, RA patients identified pain, fatigue, and independence as the most important domains of disease activity that need to be improved to reflect remission [35]. For the assessment of fatigue, the FACIT-F scale is one of the most widely used multidimensional tools [36]. The average scores in the present study are in line with those reported in a study in $557 \mathrm{RA}$ patients (28 to 32 points depending on DMARD or biologic treatment, with no significant differences) [37]. The positive treatment effect in GO-NICE is in line with a number of clinical trials in RA [37], in PsA [38], and in AS [39].

\section{Methodological considerations}

PRO are intrinsically subjective and present a range of scientific and logistical challenges. For example, in surveys in the UK, researches and trial personnel have stated substantial inconsistencies in the level of assistance given to trial participants, the timing of PRO materials' completion in relation to the clinical consultation, and the routine screening for avoidable missing data in order to maximise data quality/ minimise risk of bias [40, 41].

This study has several strengths and limitations. Strengths include the relatively large cohort of prospectively enrolled and consecutive patients with one of the three index diagnoses which were mainly treated by rheumatologists. The initial responses to GLM treatment as well as overall outcomes match the effects seen in the phase III pivotal trials what externally validates the results.

As limitations, the relatively high rate of patients who were lost to follow-up must be considered (with no information on the outcomes on these patients). Similar rates as in GO-NICE have been reported from the US-American Corrona RA Registry, where almost half of patients discontinued bDMARD therapies within 24 months [42].

All patients came from Germany which limits the generalizability of the findings to patients in other health care settings and countries. A further important limitation is the lack of a control group of patients who did not receive GLM therapy. Clinical decisions of the treating physicians may assign selected patients to GLM as compared to other treatment options, what potentially may introduce allocation or channelling bias and confound the association between treatment and outcomes [43]. Physicians and patients willing to participate in non-interventional studies such as GO-NICE may be particularly motivated or interested in science and, therefore, also be subject to selection bias. A major limitation is the lack of data concerning concomitant drug use (csDMARD) during the follow-up period that could possibly interfere with the PROs.

\section{Conclusions}

Golimumab (GLM) $50 \mathrm{mg} \mathrm{SC}$ monthly was an effective treatment in patients with RA, PsA, and AS in a real-life setting in Germany. During the 24-month observation, substantial improvement in patient-reported quality of life (EQ-5D-3L and EQ VAS), functional capacity (FFbH), and fatigue (FACIT-F) were seen in a very similar manner in all three diseases.

Acknowledgements The authors thank the participating investigators and their staff for their commitment.

Author contributions KK: concept, design, analysis, writing, interpretation. GRB: concept, design and analysis. SW: interpretation of results, critical revision of manuscript. MB-B: interpretation of results. MHT: concept, design, critical revision of manuscript.

Funding This study was sponsored by MSD Sharp \& Dohme GmbH, Haar, Germany.

\section{Compliance with ethical standards}

Ethics approval and consent to participate The study was performed in accordance with the Declaration of Helsinki and standards of Good Clinical Practice. The primary ethics approval was obtained from the 
Ethics Committee of the Ludwig-Maximilian University in Munich on 17 February 2010 (number 008-10). All patients provided written informed consent prior to participation.

\section{Consent for publication Not applicable.}

Availability of data and materials The datasets generated and/or analysed during the current study are not publicly available as they concern a proprietary product and sharing is not explicitly covered by patient consent.

Role of the sponsor The sponsor MSD Sharp \& Dohme GmbH funded this observational study and had a role in the design of the study and critical revision of the manuscript, in close collaboration with the scientific advisors of this study (co-authors). The authors confirm that there was on influence of the funding on the results and the conclusion.

Open Access This article is distributed under the terms of the Creative Commons Attribution 4.0 International License (http://creativeco mmons.org/licenses/by/4.0/), which permits unrestricted use, distribution, and reproduction in any medium, provided you give appropriate credit to the original author(s) and the source, provide a link to the Creative Commons license, and indicate if changes were made.

\section{References}

1. Weldring T, Smith SM (2013) Patient-reported outcomes (PROs) and patient-reported outcome measures (PROMs). Health Serv Insights. 6: 61-68

2. European Medicines Agency Reflection paper on the regulatory guidance for the use of health-related quality of life (HRQL) measures in the evaluation of medicinal products; EMEA/ CHMP/EWP/139391/2004 London, 27 July 2005. Internet: http://www.ema.europa.eu/ema/index.jsp?curl=pages/includes/ document/document_detail.jsp?webContentId=WC50000363 7\&mid=WC0b01ac058009a3dc. Accessed on 28 May 2018

3. Food and Drug Administration Guidance for industry: patientreported outcome measures: use in medical product development to support labeling claims. Rockville, USA. December (2009) Internet: https://www.fda.gov/downloads/drugs/guidances/ucm19 3282.pdf. Accessed on 28 May 2018

4. Bartlett SJ, Hewlett S, Bingham CO, et al (2012) Identifying core domains to assess flare in rheumatoid arthritis: an OMERACT international patient and provider combined Delphi consensus. Ann Rheum Dis 71:1855-1860

5. McInnes IB, Combe B, Burmester G (2013) Understanding the patient perspective - results of the rheumatoid arthritis: insights, strategies and expectations (RAISE) patient needs survey. Clin Exp Rheumatol 31:350-357

6. Wollenhaupt J, Ehlebracht-Koenig I, Groenewegen A, Fricke D (2013) Prioritizing the patient: optimizing therapy in rheumatoid arthritis. Results of a patient questionnaire in northern Germany. Open Access Rheumatol 5:51-67

7. Graninger W, Gross WL, Raspe H (2010) Quality of life in rheumatic diseases. Z Rheumatol 69:197

8. Smolen JS, Landewe R, Breedveld FC et al (2014) EULAR recommendations for the management of rheumatoid arthritis with synthetic and biological disease-modifying antirheumatic drugs: 2013 update. Ann Rheum Dis 73:492-509

9. Strand V, Singh JA (2010) Newer biological agents in rheumatoid arthritis: impact on health-related quality of life and productivity. Drugs 70:121-145
10. Boyce EG, Halilovic J, Stan-Ugbene O (2010) Golimumab: review of the efficacy and tolerability of a recently approved tumor necrosis factor-alpha inhibitor. Clin Ther 32:1681-1703

11. Keystone EC, Genovese MC, Klareskog L et al (2009) Golimumab, a human antibody to tumour necrosis factor $\{$ alpha given by monthly subcutaneous injections, in active rheumatoid arthritis despite methotrexate therapy: the GO-FORWARD Study. Ann Rheum Dis 68:789-796

12. Kavanaugh A, McInnes I, Mease P et al (2009) Golimumab, a new human tumor necrosis factor alpha antibody, administered every four weeks as a subcutaneous injection in psoriatic arthritis: twenty-four-week efficacy and safety results of a randomized, placebo-controlled study. Arthritis Rheum 60:976-986

13. Inman RD, Davis JC, Heijde D et al (2008) Efficacy and safety of golimumab in patients with ankylosing spondylitis: results of a randomized, double-blind, placebo-controlled, phase III trial. Arthritis Rheum 58:3402-3412

14. Braun J, Baraliakos X, Hermann KG et al (2014) The effect of two golimumab doses on radiographic progression in ankylosing spondylitis: results through 4 years of the GO-RAISE trial. Ann Rheum Dis 73:1107-1113

15. Kay J, Fleischmann R, Keystone E et al (2016) Five-year safety data from 5 clinical trials of subcutaneous Golimumab in patients with rheumatoid arthritis, psoriatic arthritis, and ankylosing spondylitis. J Rheumatol 43:2120-2130

16. Keystone EC, Genovese MC, Hall S et al (2016) Safety and efficacy of subcutaneous golimumab in patients with active rheumatoid arthritis despite methotrexate therapy: final 5-year results of the GO-FORWARD trial. J Rheumatol 43:298-306

17. Emery P, Fleischmann RM, Strusberg I et al (2015) Efficacy and safety of subcutaneous golimumab in methotrexate-naive patients with rheumatoid arthritis: 5-year results of the GOBEFORE trial. Arthritis Care Res (Hoboken) 68:744-752

18. Deodhar A, Braun J, Inman RD et al (2015) Golimumab administered subcutaneously every 4 weeks in ankylosing spondylitis: 5-year results of the GO-RAISE study. Ann Rheum Dis 74:757-761

19. Frampton JE (2017) Golimumab: a review in inflammatory arthritis. Bio Drugs 31:263-274

20. Krüger K, Burmester G, Wassenberg S, Bohl-Bühler M, Thomas M (2018) Effectiveness and safety of golimumab in patients with rheumatoid arthritis, psoriatic arthritis, and ankylosing spondylitis under real-life clinical conditions: GO-NICE study in Germany. BMJ Open 0:e021082. https://doi.org/10.1136/ bmjopen-2017-021082

21. Ruof J, Iking-Konert C, Simianer S, Burmester GR (2014) [Phase IV non-interventional studies in the treatment of rheumatoid arthritis with biologicals in Germany: real-life clinical practice data]. Zeitschrift Rheumatol 73:65-73

22. European Agency for the Evaluation of Medicinal Products (EMA) Simponi (Golimumab) Summary of Product Characteristics (SmPC). Latest renewal of authorisation 19 June 2014. Internet: http://www.ema.europa.eu. Accessed on 28 May 2018

23. Greiner W, Claes C, Busschbach JJ, von der Schulenburg JM (2005) Validating the EQ-5D with time trade off for the German population. Eur J Health Econ 6:124-130

24. Lautenschlager J, Mau W, Kohlmann T et al (1997) Comparative evaluation of a German version of the Health Assessment Questionnaire and the Hannover Functional Capacity Questionnaire. Z Rheumatol 56:144-155

25. Cella D, Lai JS, Chang CH, Peterman A, Slavin M (2002) Fatigue in cancer patients compared with fatigue in the general United States population. Cancer 94:528-538

26. Chandran V, Bhella S, Schentag C, Gladman DD (2007) Functional assessment of chronic illness therapy-fatigue scale 
is valid in patients with psoriatic arthritis. Ann Rheum Dis 66:936-939

27. Mease PJ, Gladman DD, Ritchlin CT et al (2005) Adalimumab for the treatment of patients with moderately to severely active psoriatic arthritis: results of a double-blind, randomized, placebocontrolled trial. Arthritis Rheum 52:3279-3289

28. Montan I, Löwe B, Cella D, Mehnert A, Hinz A (2018) General population norms for the functional assessment of chronic illness therapy (FACIT)-Fatigue Scale. Value Health. https://doi. org/10.1016/j.jval.2018.03.013 (in press)

29. Smolen JS, Kay J, Doyle MK et al (2009) Golimumab in patients with active rheumatoid arthritis after treatment with tumour necrosis factor alpha inhibitors (GO-AFTER study): a multicentre, randomised, double-blind, placebo-controlled, phase III trial. Lancet 374:210-221

30. Emery P, Fleischmann RM, Moreland LW et al (2009) Golimumab, a human anti-tumor necrosis factor alpha monoclonal antibody, injected subcutaneously every four weeks in methotrexate-naive patients with active rheumatoid arthritis: twenty-fourweek results of a phase III, multicenter, randomized, double-blind, placebo-controlled study of golimumab before methotrexate as first-line therapy for early-onset rheumatoid arthritis. Arthritis Rheum 60:2272-2283

31. Ware JE, Sherbourne CD (1992) The MOS 36-item short-form health survey (SF-36). I. Conceptual framework and item selection. Med Care 30:473-483

32. van der Heijde D, Deodhar A, Braun J et al (2014) The effect of golimumab therapy on disease activity and health-related quality of life in patients with ankylosing spondylitis: 2-year results of the GO-RAISE trial. J Rheumatol 41:1095-1103

33. Gaubitz M, Göttl K-H, Behmer O, Lippe R, Meng T, Löschmann P-A (2017) Etanercept is effective as monotherapy or in combination with methotrexate in rheumatoid arthritis: subanalysis of an observational study. Clin Rheumatol 36:1989-1996

34. Katz P (2017) Fatigue in rheumatoid arthritis. Curr Rheumatol Rep 19:25

35. van Tuyl LH, Sadlonova M, Hewlett S et al (2017) The patient perspective on absence of disease activity in rheumatoid arthritis: a survey to identify key domains of patient-perceived remission. Annals Rheum Dis 76:855-861
36. Mease PJ (2011) Measures of psoriatic arthritis: Tender and Swollen Joint Assessment, Psoriasis Area and Severity Index (PASI), Nail Psoriasis Severity Index (NAPSI), Modified Nail Psoriasis Severity Index (mNAPSI), Mander/Newcastle Enthesitis Index (MEI), Leeds Enthesitis Index (LEI), Spondyloarthritis Research Consortium of Canada (SPARCC), Maastricht Ankylosing Spondylitis Enthesis Score (MASES), Leeds Dactylitis Index (LDI), Patient Global for Psoriatic Arthritis, Dermatology Life Quality Index (DLQI), Psoriatic Arthritis Quality of Life (PsAQOL), Functional Assessment of Chronic Illness Therapy-Fatigue (FACIT-F), Psoriatic Arthritis Response Criteria (PsARC), Psoriatic Arthritis Joint Activity Index (PsAJAI), Disease Activity in Psoriatic Arthritis (DAPSA), and Composite Psoriatic Disease Activity Index (CPDAI). Arthritis Care Res (Hoboken) 63(11):S64-85

37. Campbell RCJ, Batley M, Hammond A, Ibrahim F, Kingsley G, Scott DL (2012) The impact of disease activity, pain, disability and treatments on fatigue in established rheumatoid arthritis. Clin Rheumatol 31:717-722

38. Strand V, Schett G, Hu C, Stevens RM (2013) Patient-reported Health-related Quality of Life with apremilast for psoriatic arthritis: a phase II, randomized, controlled study. J Rheumatol 40:1158-1165

39. Deodhar AA, Dougados M, Baeten DL et al (2016) Effect of Secukinumab on patient-reported outcomes in patients with active ankylosing spondylitis: a phase III randomized trial (MEASURE 1). Arthritis Rheumatol 68:2901-2910

40. Kyte D, Ives J, Draper H, Calvert M (2016) Current practices in patient-reported outcome (PRO) data collection in clinical trials: a cross-sectional survey of UK trial staff and management. BMJ Open 6:e012281

41. Kyte D, Ives J, Draper H, Calvert M (2016) Management of patient-reported outcome (PRO) alerts in clinical trials: a cross sectional survey. PLoS One 11:e0144658

42. Strand V, Miller P, Williams SA, Saunders K, Grant S, Kremer J (2017) Discontinuation of biologic therapy in rheumatoid arthritis: analysis from the Corrona RA registry. Rheumatol Ther 4:489-502

43. Delgado-Rodriguez M, Llorca J (2004) Bias. J Epidemiol Community Health 58:635-641 